\title{
RECRUDESCENCE IN ARTESUNATE-TREATED PATIENTS WITH FALCIPARUM MALARIA IS DEPENDENT ON PARASITE BURDEN NOT ON PARASITE FACTORS
}

\author{
WANIDA ITTARAT, AMY L. PICKARD, PANTHIP RATTANASINGANCHAN, POLRAT WILAIRATANA, \\ SORNCHAI LOOAREESUWAN, KATHRYN EMERY, JONATHAN LOW, RACHANEE UDOMSANGPETCH, AND \\ STEVEN R. MESHNICK \\ Departments of Clinical Microscopy, Faculty of Medical Technology, Mahidol University, Bangkok, Thailand; Department of \\ Epidemiology, School of Public Health, University of North Carolina, Chapel Hill, NC; Hospital for Tropical Disease, Faculty of \\ Tropical Medicine/Mahidol University, Bangkok, Thailand; Department of Epidemiology, School of Public Health, University of \\ Michigan, Ann Arbor, MI; Department of Experimental Pathobiology, Faculty of Science, Mahidol University, Bangkok, Thailand
}

Abstract. Artemisinin derivatives are first-line antimalarial drugs in Thailand. No firm evidence of clinically relevant artemisinin resistance exists. When used as monotherapy, artesunate has been associated with a high treatment failure (recrudescence) rate, which could be due to low-level artemisinin resistance. To understand the causes of recrudescence, we retrospectively studied a cohort of 104 malaria patients treated with artesunate monotherapy, 32 of whom recrudesced. There was no difference in in vitro artesunate sensitivities between 6 nonrecrudescent isolates and 16 paired admission and recrudescent isolates. Paired admission and recrudescent isolates from 10 patients were genotyped; only 3 had pfmdrl mutations. Patients with admission parasitemias $>10,000$ per $\mu$ l had a 9-fold higher likelihood of recrudescence (adjusted odds ratio) compared with patients with lower parasitemias. This study suggests (1) recrudescence after treatment with artesunate is not the result of inherent parasite resistance, and (2) admission parasitemia may be useful in choosing therapeutic options.

\section{INTRODUCTION}

Falciparum malaria has developed resistance to most current antimalarials. ${ }^{1}$ Multidrug-resistant falciparum malaria is particularly common in Southeast Asia, where it has greatly limited therapeutic options. Artemisinin and its derivatives are the only remaining drugs that are effective against severe malaria and against multidrug-resistant malaria. ${ }^{2}$ Accordingly, artesunate, a semisynthetic derivative of artemisinin, in combination with mefloquine, is now the first-line treatment of malaria in certain regions of Thailand, where it has been used widely since $1992 .^{3}$

Although different isolates of Plasmodium falciparum vary in sensitivity to artemisinin in vitro, there is currently no hard evidence for the existence of artemisinin resistance in vivo. There have been reports of patients who failed therapy with artemisinins, but when patient isolates were tested in vitro, there was no evidence for decreased parasite susceptibility to drug. ${ }^{4-6}$ Artemisinin-resistant murine malarias have been generated and characterized. ${ }^{7}$ There are 3 possible reasons why clinically important artemisinin resistance has not yet appeared. First, artemisinin derivatives now are usually used in combination with other drugs, and combination chemotherapy slows down the development of resistance. ${ }^{1}$ Second, artemisinins have gametocytocidal activity, and this may inhibit the transmission of drug-resistant strains from person to person. ${ }^{8}$ Third, artemisinin derivatives have short half-lives, and drugs with short half-lives tend to be less prone to developing resistance. ${ }^{9}$

When used as monotherapy, artemisinin derivatives are associated with high rates of recrudescence. ${ }^{10}$ These recrudescences could be due to either parasite factors (resistance) or host factors. ${ }^{11}$ To determine why patients have recrudescence after treatment with artemisinin derivatives, we retrospectively analyzed data and stored parasite isolates from patients treated with artesunate monotherapy at the Bangkok Hospital for Tropical Diseases, Thailand, investigating host-related and parasite-related factors.

\section{MATERIALS AND METHODS}

This study was approved by the Ethics Committee of Mahidol University. Unless specified, all chemicals used in the present study were purchased from Sigma (St. Louis, MO).

Patient recruitment, treatment, and evaluation. Of 320 patients with falciparum malaria admitted during the period April 1998-March 1999 to the Bangkok Hospital for Tropical Diseases, Thailand, 150 patients were treated with artesunate tablets (600 mg total dose in 3 days; Guilin No. 1 Factory, Guangxi, China, registered for use in Thailand). Blood was collected routinely from all malaria patients. Inclusion criteria were as follows: had a case of acute uncomplicated falciparum malaria, were $>18$ years old, had no treatment with artemisinin derivatives during the previous 2 weeks, and gave informed consent. There were 104 patients who met the inclusion criteria for this study. Patients remained in the hospital for 28 days and had daily blood smears. Blood samples were taken before treatment on the day of admission and at the time of recrudescence and were stored in liquid nitrogen. Routine blood chemistry and hematologic parameters were determined automatically using a Boehringer Mannheim Hitachi 917 (Boehringer Mannheim, Indianapolis, IN) and Gen S (Coulter, Hialeah, FL).

The freezing solution for cryopreservation of parasites was prepared by first filtering a solution of either sorbitol or glycerol $(4.2 \%)$ in $0.9 \%$ sodium chloride, through a $0.45-\mu \mathrm{m}$ filter (New Bedford, MA). This solution was mixed with $99 \%$ glycerol to make a solution containing 3\% sorbitol or glycerol, $0.65 \%$ sodium chloride, and $28 \%$ glycerol. Before cryopreservation, ring-stage patient blood samples were centrifuged at $400 \times \mathrm{g}$ for 7 minutes at $4^{\circ} \mathrm{C}$. The plasma was discarded, and the cell pellet was mixed with an equal volume of freezing solution. The mixture was divided into $0.5-\mathrm{ml}$ aliquots, frozen, and stored in liquid nitrogen.

Parasite density was obtained from thin blood smears: 1,000 erythrocytes were examined, and the number of these infected with plasmodia yielded the percent parasitemia. Para- 
sitemias were calculated as parasites per microliter of blood (multiplying percent parasitemia and red blood cell [RBC] counts).

Parasite drug sensitivity tests. Blood samples were thawed, and the in vitro culture of the blood stage malaria parasite was performed by candle jar method as described previously using human $\mathrm{AB}$ serum. ${ }^{12}$ Aliquots were removed and thawed underwater at room temperature. An equal volume of $3.5 \%$ sodium chloride was added, and the mixture was centrifuged at $400 \times \mathrm{g}$ for 7 minutes at $4{ }^{\circ} \mathrm{C}$. The pellet was washed 3 times with stock RPMI 1640 medium and resuspended in RPMI. Parasites were cultured in a candle jar at $37^{\circ} \mathrm{C}$ until parasitemia reached $0.4 \%$ to $0.6 \%$ and were used for drug sensitivity assays. Individual isolates were not cultured for equal duration because culture time depended on starting parasitemia level; generally, no cultures were $>1$ month. Sensitivities to dihydroartemisinin were determined by the microdilution method using tritiated hypoxanthine incorporation. ${ }^{13} \mathrm{~A}$ 2 -fold serial dilution of dihydroartemisinin was prepared in a 96-well plate to which a parasitized RBC suspension, mostly at the ring stage, was added. The plate was incubated at $37^{\circ} \mathrm{C}$ for 24 hours in a candle jar. Tritiated hypoxanthine (Amersham, Arlington Heights, IL) was added, after which the plate was incubated for another 18-20 hours. The infected cell suspension was harvested (Nunc Cell Harvester 8; Nalge Nunc International, Rochester, NY) and hypoxanthine incorporation was determined by a scintillation counter (Tricarb 2700 TR; Packard Company).

Hemoglobin typing and measurement of glucose-6phosphate dehydrogenase. Hemoglobin type was analyzed by cellulose acetate electrophoresis according to the method described by the manufacturer (Helena Laboratories, Beaumont, TX). Hemoglobins are separated at a $\mathrm{pH}$ of 8.6 on a cellulose acetate plate, then stained with Ponceau S. For some patients, the chromatogram could not distinguish conclusively between hemoglobin $\mathrm{A}_{2}$ and hemoglobin E. In those cases, identification was based on the result of anion exchange chromatography (see later).

The quantitation of hemoglobin $\mathrm{A}_{2}$ by anion exchange chromatography was performed according to the method described by the manufacturer (Helena Laboratories, Beaumont, TX). Under these conditions, hemoglobin $\mathrm{A}_{2}$ was eluted while normal, and other abnormal hemoglobins were retained by the resin. The hemoglobin $\mathrm{A}_{2}$ fraction was compared with a total hemoglobin fraction by determining the absorbance and calculating for the percentage of hemoglobin $\mathrm{A}_{2}$. If the hemoglobin $\mathrm{A}_{2}$ was $<3.5 \%$, then the inconclusive band on cellulose acetate was considered to be hemoglobin $\mathrm{E}$.

Glucose-6-phosphate dehydrogenase (G6PD) deficiency was assessed by fluorescent qualitative spot test. ${ }^{14}$ The reaction mixture containing glucose-6-phosphate (G-6-P), triphosphopyridine nucleotide (TPN), saponin, and oxidized glutathione (GSSG) in potassium phosphate buffer was added to the blood samples. After incubation at $37^{\circ} \mathrm{C}$ for 3 minutes, the mixture was spotted onto a Whatman no. 1 filter paper. In the presence of G6PD, the substrate, G-6-P, was oxidized to 6-phosphogluconate, while TPN was reduced (TPNH), resulting in a fluorescent spot under long UV light.

Analysis of strains for prmdr1 polymorphisms. Parasitized RBCs were harvested from culture when $>80 \%$ of the parasites were trophozoites and stored at $-20^{\circ} \mathrm{C}$. DNA was extracted from the pellets using QIAamp DNA minikits
(Qiagen, Valencia, CA). Four regions of the pfmdr1 gene were polymerase chain reaction (PCR) amplified by modifications of previously published methods. ${ }^{15,16}$ Basically, we performed single-round PCR using the flanking primers described in these reports. For all 4 reactions, we used the PCR conditions described as PCR 184. We did not perform the second-round, however, allele-specific reaction. Instead, amplified DNA was purified and sequenced. ${ }^{17}$

Statistical analysis. SAS version 8.2 (Cary, NC) was used to construct logistic regression models, with recrudescence as the dependent variable. Variables representing patient characteristics and laboratory test results were analyzed individually first, then multivariate models were constructed to control for important covariates. For simplicity, the continuous variable representing number of parasites per microliter of blood was categorized into a dichotomous variable. The cutpoint for the dichotomous high/low parasitemia was set at 10,000 parasites/ $\mu$ l blood, which has been used in the literature. ${ }^{18}$ SAS also was used to conduct survival analysis for modeling time to recrudescence.

\section{RESULTS}

Recrudescent rate after artesunate administration. A total of 104 patients with falciparum malaria were admitted to the malaria ward of the Hospital for Tropical Diseases and treated with $600 \mathrm{mg}$ of artesunate for 3 days. All patients remained in the hospital for 28 days, and 32 patients recrudesced during their hospital stays (29\%).

Characteristics of recrudescent and nonrecrudescent parasite isolates. Of all the available blood samples, in vitro parasite cultures were obtained successfully from 6 nonrecrudescent patients and 16 recrudescent patients (on admission and during the episode of recrudescence) (Figure 1). Because artesunate is rapidly converted to dihydroartemisinin in vivo, $50 \%$ inhibitory concentrations $\left(\mathrm{IC}_{50}\right)$ for dihydroartemisinin were determined for each parasite isolate. A Wilcoxon signed rank test (for recrudescent versus nonrecrudescent patients) and a paired $t$-test (for admission versus recrudescent isolates) were used to evaluate differences in $\mathrm{IC}_{50}$. There were no significant differences in $\mathrm{IC}_{50}$ for parasites from recrudescent patients on admission $(0.57 \pm 0.44 \mathrm{ng} / \mathrm{ml}$; range, $0.17-1.56$ $\mathrm{ng} / \mathrm{ml})$ or recrudescence $(0.615 \pm 0.534 \mathrm{ng} / \mathrm{ml}$; range, $0.12-$ $1.75)$ or from nonrecrudescent patients $(0.45 \pm 0.22 \mathrm{ng} / \mathrm{ml}$; range, $0.2-0.86 \mathrm{ng} / \mathrm{ml})$.

Because all samples were frozen in liquid nitrogen before $\mathrm{IC}_{50}$ determination, the effects of freezing and thawing on $\mathrm{IC}_{50}$ were tested using the laboratory strain (K1). K1 was frozen and thawed under similar conditions $(\mathrm{n}=5)$. The $\mathrm{IC}_{50}$ was $0.38 \pm 0.09 \mathrm{ng} / \mathrm{ml}$ before and $0.30 \pm 0.07 \mathrm{ng} / \mathrm{ml}$ after freeze-thawing. There was no statistically significant difference using a paired $t$-test $(P=0.87)$, indicating that freezing, thawing, and reculturing did not affect the determination of $\mathrm{IC}_{50}$.

In previous reports, $p$ fmdr1 mutations have been associated with altered in vitro sensitivity to artemisinins. ${ }^{19-21} \mathrm{Se}$ quence polymorphisms in $p f m d r 1$ that may decrease sensitivity have been reported at several codon positions: N86Y, Y184F, S1034C, N1042D, and D1246Y. For genotyping experiments, new cultures had to be established from cryopreserved stabilates. Cultures were obtained successfully from 10 


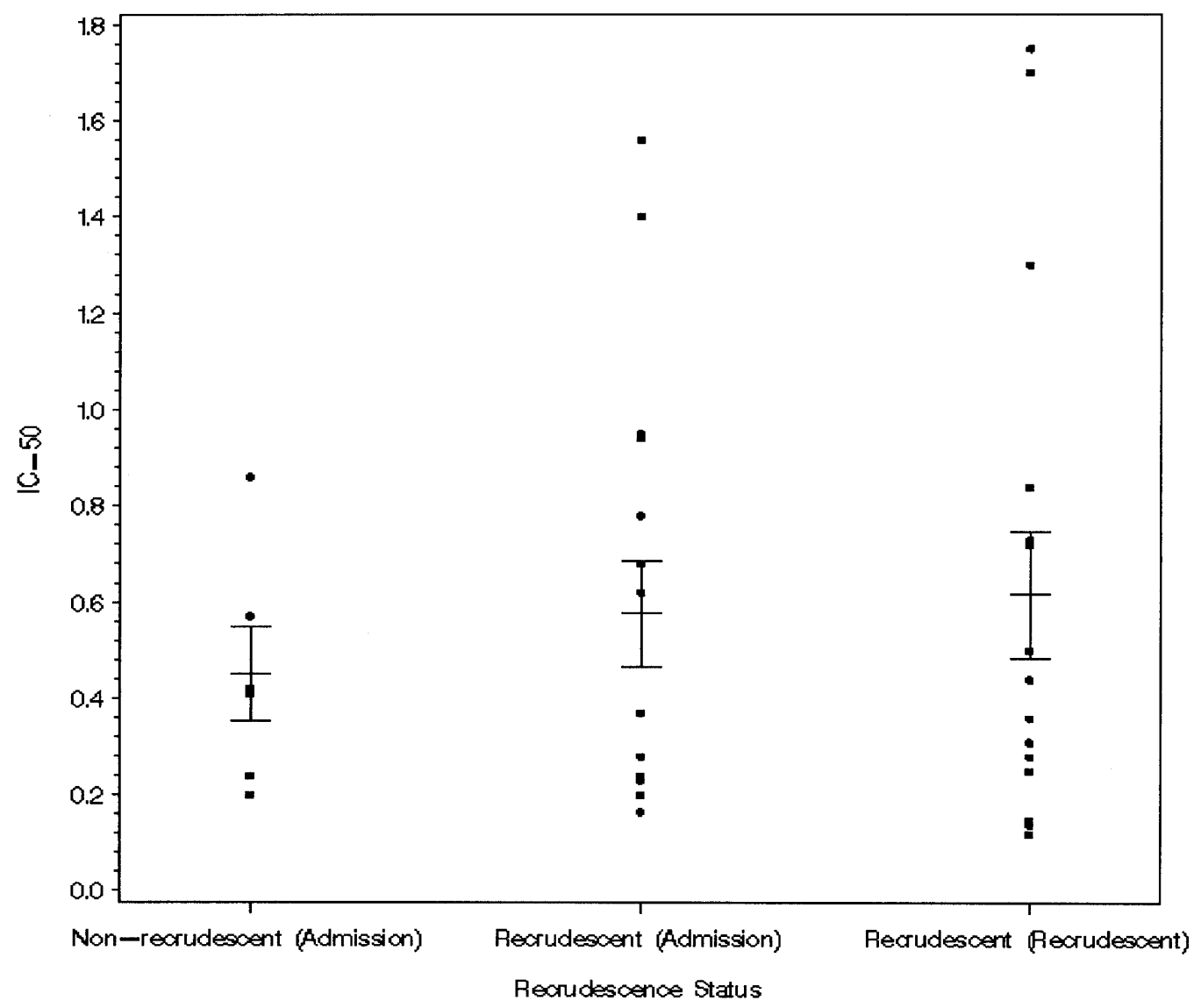

FIGURE 1. Dihydroartemisinin $50 \%$ inhibitory concentrations $\left(\mathrm{IC}_{50}\right)$ of cultured patient isolates (in $\mathrm{ng} / \mathrm{ml}$ ).

recrudescent patients (from isolates obtained on admission and during their recrudescent episodes). We PCR amplified and sequenced the $p f m d r l$ genes from the admission and recrudescent cultures from these 10 patients. Initial and recrudescent cultures from 7 of 10 patients had wild-type $p f m d r 1$ genotypes. Isolates from only 3 patients had mutations. In 2 of these 3 patients, isolates showed the same mutations before and after treatment (one had the N1042D mutation and the other was Y184F). In 1 of the 3 patients, the pretreatment isolate had a Y184F mutation that was not found in the posttreatment strain, suggesting that the original infection may have been polyclonal and that the nonmutant strain was responsible for the recrudescence.

Characteristics of recrudescent and nonrecrudescent patients. The characteristics of the 32 recrudescent patients and 72 nonrecrudescent patients are shown in Table 1 . The ages of patients with and without recrudescence were similar $(25.9 \pm$ 6.8 years and $24.9 \pm 8.0$ years $)$. Both groups were predominantly male $(69 \%$ of recrudescent patients and $63 \%$ of nonrecrudescent patients). None of the patients were homozygous for thalassemia. Of 103 patients, 18 (17.3\%) were heterozygous for hemoglobin $\mathrm{E}$ ( $\mathrm{Hb} \mathrm{EA}$ ), a type of $\beta$-thalassemia, and of 104 patients, 9 (8.7\%) had G6PD deficiency. No other abnormal hemoglobin bands were detected. There were no significant differences between the 2 groups in admission blood chemistries, mean corpuscular volume, mean corpuscular hemoglobin concentration, or platelet counts or in the percentage of patients with G6PD deficiencies or thalassemic trait.
There were several significant differences between patients who had recrudescence and patients who did not. First, the recrudescent patients had higher percent parasitemias on admission $(2.65 \%$ versus $0.45 \%$ in nonrecrudescent patients). Second, recrudescent patients had significantly higher RBC counts, hemoglobin levels, and hematocrits (Table 1). Together, these data suggest that patients with higher parasite burdens were more likely to have recrudescence. When the number of parasites per microliter of blood was calculated, the difference was highly significant $(P=0.0035)$. The only other variable that was significantly different between recrudescent and nonrecrudescent patients was the white blood cell (WBC) count; recrudescent patients had significantly higher WBC counts than nonrecrudescent patients (6.6 versus 5.7). The odds ratio (OR) for recrudescence for patients having WBC counts $>5.85$ (the median) was 3.08 (95\% confidence interval [CI], 1.27-7.44).

Logistic regression was done to confirm the association between recrudescence and factors found to be significant in univariate analyses, including RBC count, hematocrit, hemoglobin level, and percent parasitemia and parasitemia (num-

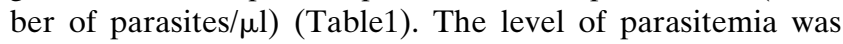
represented by a dichotomous variable for high/low level of parasitemia, with 10,000 parasites/ $\mu l$ as the cut-point. ${ }^{18}$ (Similar results were found when the median, 12,150, was used as the cut-point.) Many of these factors are either closely related (RBC count, hemoglobin, and hematocrit) or indirectly related (i.e., RBC count $\times$ percent parasitemia $=$ parasitemia). The strongest univariate association was with parasitemia, 
TABLE 1

Characteristics of recrudescent and nonrecrudescent patients

\begin{tabular}{|c|c|c|c|}
\hline & $\begin{array}{l}\text { Recrudescent } \\
(\mathrm{n}=32)\end{array}$ & $\begin{array}{c}\text { Nonrecrudescent } \\
(\mathrm{n}=72)\end{array}$ & $P$ value* \\
\hline Age of infected patient (yr) & $25.9(13-41)$ & $26.9(14-49)$ & NS \\
\hline Sex of infected patients (M:F) & $22: 10$ & $45: 27$ & NS \\
\hline Percent parasitemia on admission & $2.65(0.02-20.20)$ & $0.45(<0.01-0.40)$ & 0.0106 \\
\hline Parasitemia (per $\mu \mathrm{l})$ & $116,000(590-776,000)$ & $19,000(30-174,000)$ & 0.0035 \\
\hline Parasitemia, $>10,000$ per $\mu \mathrm{l}$ (no. [\%]) & $28(87.5)$ & $30(41.7)$ & $<0.001$ \\
\hline Samples with G6PD deficiency (\%) & $2(6.3)$ & $\begin{array}{l}7(9.3) \\
(\mathrm{n}=70)\end{array}$ & $\mathrm{NS} \dagger$ \\
\hline Hb EA-containing samples (\%) & $3(9.4)$ & $\begin{array}{l}16(21.1) \\
(\mathrm{n}=71)\end{array}$ & $\mathrm{NS} \dagger$ \\
\hline AST (IU/L) & $71.3(20-315)$ & $53.0(15-266)$ & NS \\
\hline ALT (IU/L) & $57.9(5-266)$ & $53.7(8-211)$ & NS \\
\hline BUN (mg/dL) & $19.25(3.50-91.20)$ & $13.6(0.55-62.00)$ & NS \\
\hline BUN >20 mg/dL (no. [\%]) & $9(28.1)$ & $6(8.3)$ & 0.0284 \\
\hline Alkaline phosphatase (IU/L) & $101.5(53.0-228.8)$ & $109.8(34.1-279.6)$ & NS \\
\hline RBC ( 1 million per $\mu \mathrm{l})$ & $4.6(2.6-6.5)$ & $4.2(2.2-6.2)$ & 0.030 \\
\hline Hemoglobin (\%) & $12.3(7.8-15.7)$ & $11.1(6.4-16.8)$ & 0.022 \\
\hline Hematocrit (\%) & $37(22-48)$ & $34(19-50)$ & 0.020 \\
\hline MCV (fL) & $82(64-96)$ & $\begin{array}{r}82(57-98) \\
(\mathrm{n}=71)\end{array}$ & NS \\
\hline $\mathrm{MCHC}(\%)$ & $32.9(31-35)$ & $\begin{array}{r}33(30-35) \\
(\mathrm{n}=71)\end{array}$ & NS \\
\hline WBC $(1,000$ per $\mu l)$ & $6.6(1.9-13.2)$ & $5.7(2.1-11.2)$ & 0.053 \\
\hline Platelets $(1,000$ per $\mu \mathrm{l})$ & $\begin{array}{c}90(11-243) \\
(\mathrm{n}=30)\end{array}$ & $109(11-358)$ & NS \\
\hline
\end{tabular}

with an OR of 9.80 for the dichotomous variable $(95 \% \mathrm{CI}$, 3.11-30.88).

The association of WBC count with recrudescence also was confirmed with logistic regression (OR, 1.24; 95\% CI, 1.001.53). The OR indicates the increase in odds of recrudescence for each increase of $1,000 / \mu \mathrm{L}$ in WBC count. When controlling for parasitemia, the OR for recrudescence with increasing WBC count remained approximately the same but lost significance (OR, 1.20; 95\% CI, 0.94-1.53). To explore the issue of host $\mathrm{WBC}$ count and recrudescent malaria, the types and amounts of WBC were determined. WBC types measured included eosinophils, basophils, monocytes, lymphocytes, and banded and segmented neutrophils. The measurements of each of these WBCs were analyzed as continuous variables to test for an association with recrudescence (trend) using logistic regression.

In univariate regression of WBC types, eosinophil, basophil, monocyte, and lymphocyte levels had no association with recrudescence (data not shown). Band neutrophils had an OR of 1.01 (95\% CI, 1.00-1.02), and segmented neutrophils had an OR of 1.00 (95\% CI, 1.00-1.01). When controlling for parasitemia, segmented neutrophils had an OR of 1.00 (95\% CI, 1.00-1.01). The WBC types individually did not have a meaningful association with recrudescence.

We then evaluated whether time to recrudescence was associated with any particular host factor. Survival analyses were performed for all of the variables in Table 1 versus time to recrudescence. Significant associations were found for many of the same variables as in bivariate analysis (parasitemia, RBC count, hemoglobin level), which suggest that time to recrudescence depends on the parasite burden. We also found that time to recrudescence depended on blood urea nitrogen (BUN) level. Figure 2 shows survival curves of re- crudescent patients categorized into normal $(\leq 20 \mathrm{mg} / \mathrm{dl})$ and high $(>20 \mathrm{mg} / \mathrm{dl})$ categories. The hazard ratio decreased significantly with the higher level of BUN $(P=0.028)$. Patients with high BUN levels (and poorer renal function) had recrudescence later than patients with low BUN levels.

\section{DISCUSSION}

To our knowledge, this is the first study to look at risk factors for failure by artemisinin monotherapy. The predominant risk factor for recrudescence seems to be a high parasite burden. Among patients with recrudescences, those with poorer renal function tended to have recrudescence later. There were no differences in $\mathrm{IC}_{50}$ values between strains isolated from recrudescent and nonrecrudescent patients on admission or between admission and recrudescent strains from patients having recrudescence. Few mutations were found in the $p f m d r 1$ gene. These data suggest that artemisinin failures were not due to drug-resistant parasites.

In this study, $31 \%$ of patients recrudesced during the 28 day observation period. Previous studies showed recrudescent rates after treatment with various regimens of artemisinin derivatives of $3-50 \% .{ }^{10}$ Longer periods of treatment are associated with lower recrudescence rates. Combination with another drug, such as mefloquine, also reduces the appearance of recrudescence. In some instances, reported recrudescence rates may be artifactually high because reinfections might be mistaken for recrudescences. In this study, however, none of the 32 recrudescent patients were cases of reinfections because all of the patients remained in the hospital in Bangkok for 28 days, where they had no exposure to infected mosquitoes, and had recrudescence during that time. 


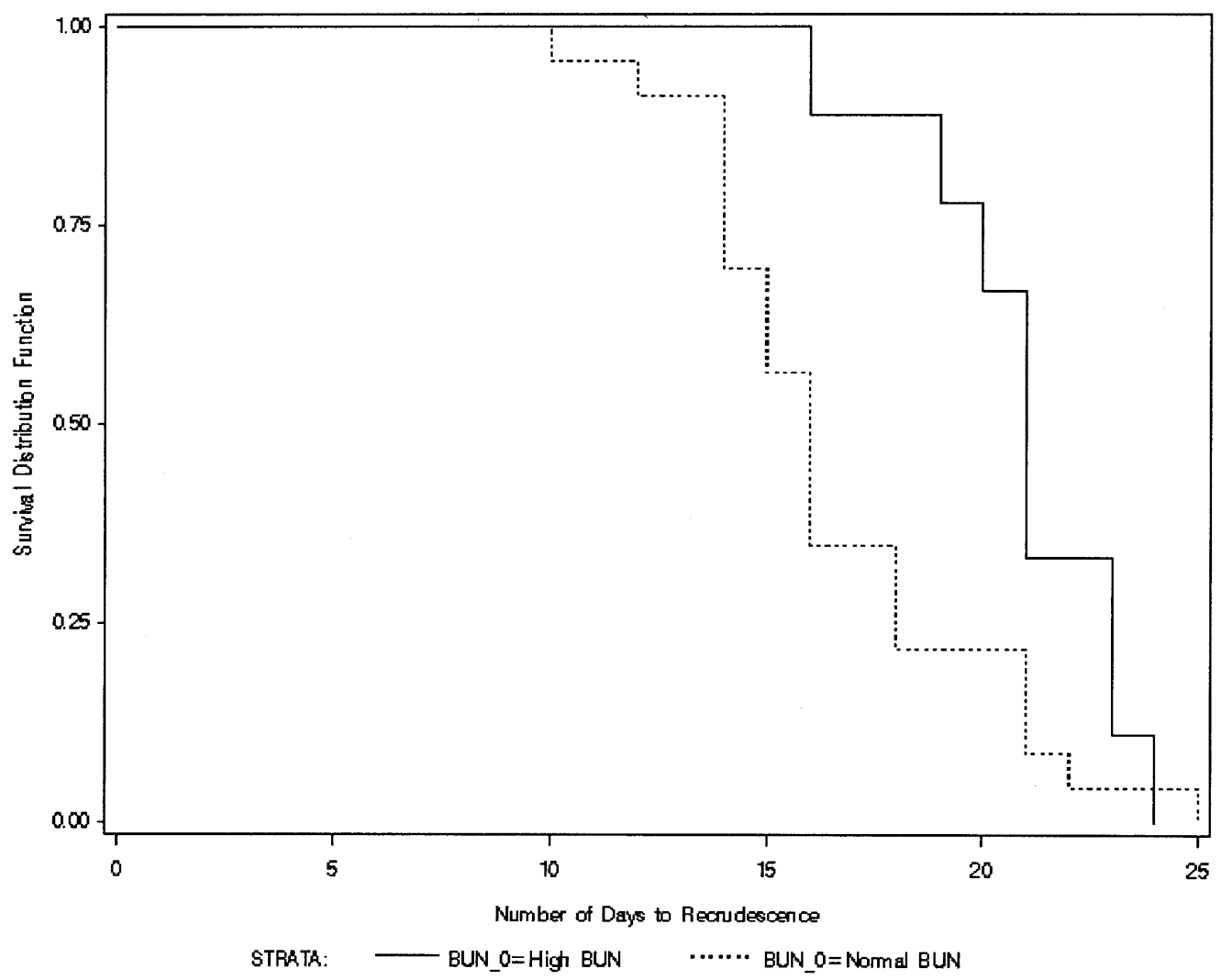

FIGURE 2. Effects of renal function on time to recrudescence. Survival curve of recrudescent patients with normal blood urea nitrogen (BUN) levels ( $\leq 20 \mathrm{mg} / \mathrm{dl}, n=23)$ (dotted line) and high BUN levels $(>20 \mathrm{mg} / \mathrm{dl}, n=9)$ (solid line).

As shown in Table 1, the parasite burdens (parasites/ $\mu$ ) on the day of admission of recrudescent patients were significantly higher than those of nonrecrudescent patients. Patients with parasitemias $>10,000$ parasites $/ \mu$ l of blood had 9.8 times the odds of recrudescence than patients with parasitemias $<10,000$ parasites/ $\mu l$. These data are consistent with previous studies on quinine ${ }^{22}$ and mefloquine, ${ }^{23}$ which also showed that recrudescent patients tended to have higher parasitemias before treatment than nonrecrudescent patients. A previous study of artemisinin monotherapy also found higher recrudescent rates with higher parasitemia; however, the association was not as strong as the one reported here. ${ }^{24}$ Higher admission parasite burdens could predispose to recrudescence by increasing the likelihood of a parasite escaping destruction.

The range of in vitro $\mathrm{IC}_{50}$ values for dihydro-artemisinin found here $(0.14-2.0 \mathrm{ng} / \mathrm{ml})$ are consistent ranges previously reported for Thai isolates $\left(0.24-8.1 \mathrm{ng} / \mathrm{ml}^{25,26}\right)$. The data presented here indicate that recrudescence after artesunate treatment was not due to the development of parasite drug resis-

TABLE 2

Odds ratio (OR) for recrudescence, crude and adjusted for age, sex, and other variables in the model

\begin{tabular}{lcccc}
\hline \multicolumn{1}{c}{ Variable } & Crude OR & \multicolumn{1}{c}{$95 \% \mathrm{CI}$} & Adjusted OR & \multicolumn{1}{c}{$95 \% \mathrm{CI}$} \\
\hline Parasitemia* & 9.80 & $3.11-30.88$ & 8.94 & $2.56-28.96$ \\
WBC & 1.24 & $1.00-1.53$ & 1.20 & $0.94-1.53$ \\
Hemoglobin & 1.23 & $1.03-1.48$ & 1.14 & $0.94-1.39$ \\
\hline
\end{tabular}

$\mathrm{CI}=$ confidence interval; $\mathrm{WBC}=$ white blood cells.

$*$ Dichotomous variable, cutpoint $=10,000$ parasites per $\mu$ l blood. tance. This finding is consistent with in vitro studies on recrudescence. $^{27}$

An association was found between high BUN level and time to recrudescence. Poor renal function may be associated with later recrudescence, perhaps because it reduces renal clearance of the drug, allowing the drug to remain at therapeutic levels in the plasma for longer periods. A longer duration of therapy may delay or prevent recrudescence; however, another study did not find longer duration of artesunate therapy to reduce recrudescence rates. ${ }^{24}$

One implication of these data is the possibility of offering longer or different therapies to patients depending on their initial parasite burdens. Admission parasitemias might be useful in helping clinicians choose therapeutic regimens.

Received May 7, 2002. Accepted for publication September 30, 2002.

Acknowledgments: We are grateful to the patients for their cooperation. We thank Donglin Zeng (Department of Biostatistics, University of North Carolina School of Public Health) for statistical help.

Financial support: This work was supported by grants from the U.S. National Institutes of Health (R21 AI45426 and R01 AI51320).

Authors' addresses: Wanida Ittarat and Panthip Rattanasinganchan, Department of Clinical Microscopy, Faculty of Medical Technology, Mahidol University, Bangkok, 10700, Thailand, Telephone: 66-24197169, Fax: 66-2-4124110, E-mail: mtwit@mucc.mahidol.ac.th. Amy L. Pickard, Department of Epidemiology, University of North Carolina School of Public Health, Chapel Hill, NC 27599, Telephone: 919-968-9651, E-mail: apickard@email.unc.edu. Polrat Wilairatana and Sornchai Looareesuwan, Department of Clinical Tropical Medicine and Bangkok Hospital for Tropical Diseases, Faculty of Tropical Medicine, Mahidol University, Bangkok, 10700, Thailand, Tele- 
phone: 66-2-2469000, Fax: 66-2-2469013. Kathryn Emery, c/o Foxman Research Group, Room 2038 University of Michigan School of Public Health, 109 S. Observatory, Ann Arbor, MI 48109, Fax: 734-9366732, E-mail: emeryk@umich.edu. Jonathan Low, 1500 E. Medical Center Drive, Box 0944, Dock 5, 6431 CCGC, Ann Arbor, MI 48109, Telephone: 734-936-0876, Fax: 734-647-9271, E-mail: jlowz@umich. edu. Rachanee Udomsangpetch, Department of Experimental Pathobiology, Faculty of Science, Mahidol University, Bangkok, 10700, Thailand, Telephone: 66-2-2461358, Fax: 66-2-2477050. Steven R Meshnick, Department of Epidemiology, University of North Carolina School of Public Health, Chapel Hill, NC 27599-7435, Telephone: 919-966-7414, Fax: 919-966-2089, E-mail: meshnick@unc.edu

Reprint requests: Steven R. Meshnick, Department of Epidemiology, University of North Carolina School of Public Health, Chapel Hill NC 27599-7435, Telephone: 919-966-7414, Fax: 919-966-2089, E-mail: meshnick@unc.edu

\section{REFERENCES}

1. White NJ, Nosten F, Looareesuwan S, Watkins WM, Marsh K, Snow RW, Kokwaro G, Ouma J, Hien TT, Molyneux ME, Taylor TE, Newbold CI, Ruebush TK, 2nd, Danis M, Greenwood BM, Anderson RM, Olliaro P, 1999. Averting a malaria disaster. Lancet 353: 1965-1967.

2. World Health Organization, 1988. The Use of Artemisinin and Its Derivatives as Antimalarial Drugs: Report of a Joint CTD/ $D M P / T D R$ Informal Consultation. Geneva: WHO.

3. Wongsrichanalai C, Pickard AL, Wernsdorfer WH, Meshnick SR, 2002. Epidemiology of drug-resistant malaria. Lancet Infect Dis 2: 209-218.

4. Sahr F, Willoughby VR, Gbakima AA, Bockarie MJ, 2001. Apparent drug failure following artesunate treatment of Plasmodium falciparum malaria in Freetown, Sierra Leone: four case reports. Ann Trop Med Parasitol 95: 445-449.

5. Luxemburger C, AB, Silamut K, Nosten F, van Vugt M, Gimenez F, Chongsuphajaisiddhi T, White NJ, 1998. Two patients with falciparum malaria and poor in vivo responses to artesunate. Trans $R$ Soc Trop Med Hyg 92: 668-669.

6. Karnad DC, Kanbur A, Kamtekar KD, Kshirsagar NA, 2000. Probable resistance to parenteral artemether in Plasmodium falciparum: case reports from Mumbai (Bombay), India. Ann Trop Med Parasitol 94: 519-520.

7. Walker DJ, Pitsch JL, Peng MM, Robinson BL, Peters W, Bhisutthibhan J, Meshnick SR, 2000. Mechanisms of artemisinin resistance in the rodent malaria pathogen Plasmodium yoelii. Antimicrob Agents Chemother 44: 344-347.

8. Price RN, Nosten F, Luxemburger C, ter Kuile FO, Paiphun L, Chongsuphajaisiddhi T, White NJ, 1996. Effects of artemisinin derivatives on malaria transmissibility. Lancet 347: 1654-1658.

9. Nzila AM, Nduati E, Mberu EK, Hopkins Sibley C, Monks SA, Winstanley PA, Watkins WM, 2000. Molecular evidence of greater selective pressure for drug resistance exerted by the long-acting antifolate Pyrimethamine/Sulfadoxine compared with the shorter-acting chlorproguanil/dapsone on Kenyan Plasmodium falciparum. J Infect Dis 181: 2023-2028.

10. Meshnick SR, Taylor TE, Kamchonwongpaisan S, 1996. Artemisinin and the antimalarial endoperoxides: from herbal remedy to targeted chemotherapy. Microbiol Rev 60: 301-315.

11. White NJ, 1997. Assessment of the pharmacodynamic properties of antimalarial drugs in vivo. Antimicrob Agents Chemother 41: 1413-1422.

12. Trager W, Jensen JB, 1976. Human malaria parasites in continuous culture. Science 193: 673-675.

13. Desjardins RE, Canfield CJ, Haynes JD, Chulay JD, 1979. Quan- titative assessment of antimalarial activity in vitro by a semiautomated microdilution technique. Antimicrob Agents Chemother 16: 710-718.

14. Pujades A, Lewis M, Salvati AM, Miwa S, Fujii H, Zarza R, Alvarez R, Rull E, Corrons JL, 1999. Evaluation of the blue formazan spot test for screening glucose 6 phosphate dehydrogenase deficiency. Int J Hematol 69: 234-236.

15. Adagu IS, Dias F, Pinheiro L, Rombo L, do Rosario V, Warhurst DC, 1996. Guinea Bissau: association of chloroquine resistance of Plasmodium falciparum with the Tyr86 allele of the multiple drug-resistance gene Pfmdr1. Trans R Soc Trop Med Hyg 90: 90-91.

16. Grobusch MP, Adagu IS, Kremsner PG, Warhurst DC, 1998. Plasmodium falciparum: in vitro chloroquine susceptibility and allele-specific PCR detection of Pfmdr1 Asn86Tyr polymorphism in Lambarene, Gabon. Parasitology 116: 211-217.

17. Lane BR, Ast JC, Hossler PA, Mindell DP, Bartlett MS, Smith JW, Meshnick SR, 1997. Dihydropteroate synthase polymorphisms in Pneumocystis carinii. J Infect Dis 175: 482-485.

18. McElroy PD, ter Kuile FO, Lal AA, Bloland PB, Hawley WA, Oloo AJ, Monto AS, Meshnick SR, Nahlen BL, 2000. Effect of Plasmodium falciparum parasitemia density on hemoglobin concentrations among full-term, normal birth weight children in western Kenya, IV. The Asembo Bay Cohort Project. Am J Trop Med Hyg 62: 504-512.

19. Duraisingh MT, Roper C, Walliker D, Warhurst DC, 2000. Increased sensitivity to the antimalarials mefloquine and artemisinin is conferred by mutations in the pfmdr1 gene of Plasmodium falciparum. Mol Microbiol 36: 955-961.

20. Reed MB, Saliba KJ, Caruana SR, Kirk K, Cowman AF, 2000. Pgh1 modulates sensitivity and resistance to multiple antimalarials in Plasmodium falciparum. Nature 403: 906-909.

21. Price R, Cassar C, Brockman A, Duraisingh M, van Vugt M, White NJ, Nosten F, Krishna S, 1999. The pfmdr1 gene is associated with a multidrug-resistant phenotype in Plasmodium falciparum from the Western border of Thailand. Antimicrob Agents Chemother 12: 2943-2949.

22. Sokhna CS, Rogier C, Dieye A, Trape JF, 2000. Host factors affecting the delay of reappearance of Plasmodium falciparum after radical treatment among a semi-immune population exposed to intense perennial transmission. Am J Trop Med Hyg 62: $266-270$.

23. ter Kuile FO, Luxemburger C, Nosten F, Thwai KL, Chongsuphajaisiddhi T, White NJ, 1995. Predictors of mefloquine treatment failure: a prospective study of 1590 patients with uncomplicated falciparum malaria. Trans $\mathrm{R}$ Soc Trop Med Hyg 89: 660-664.

24. Giao PT, Binh TQ, Kager PA, Long HP, Van Thang N, Van Nam N, de Vries PJ, 2001. Artemisinin for treatment of uncomplicated falciparum malaria: is there a place for monotherapy? Am J Trop Med Hyg 65: 690-695.

25. Wongsrichanalai C, Wimonwattrawatee T, Sookto P, Laoboonchai A, Heppner DG, Kyle DE, Wernsdorfer WH, 1999. In vitro sensitivity of Plasmodium falciparum to artesunate in Thailand. Bull World Health Organ 77: 392-398.

26. Brockman A, Price RN, van Vugt M, Heppner DG, Walsh D, Sookto P, Wimonwattrawatee T, Looareesuwan S, White NJ, Nosten F, 2000. Plasmodium falciparum antimalarial drug susceptibility on the north-western border of Thailand during five years of extensive use of artesunate-mefloquine. Trans $R$ Soc Trop Med Hyg 94: 537-544.

27. Nakazawa S, Kanbara H, Aikawa M, 1995. Plasmodiumfalciparum-recrudescence of parasites in culture. Exp Parasitol 81: 556-563. 